\title{
IMPACT OF WORK FROM HOME ON EMPLOYEES
}

\section{REENA DINESHKUMAR VARSHA PATEL}

\author{
Research Scholar, Madurai Kamaraj University, Madurai, India.
}

\begin{abstract}
In this paper I have provided an analysis of the effects of WFH (Work from home) in IT services company. The company abruptly switched all employees from WFO to WFH in March 2020, in response to the largely unanticipated pandemic shock. WFH also affected working patterns in substantial ways. Employees spent more time participating in various types of meetings, but less time in personal meetings with their manager or receiving coaching. They engaged in fewer contacts with colleagues inside and outside of the firm.
\end{abstract}

Keywords: work from home, job satisfaction, Productivity, IT services

\section{INTRODUCTION}

Working from Home [WFH] has been rising for years, as more occupations use computers and telecom- munications, more people have reliable home Internet connections, and more families have both parents working full time. The Covid-19 pandemic accelerated this process by forcing a large fraction of the global workforce to switch to WFH at least temporarily. Compared to Working from the Office [WFO], WFH has the potential to reduce commute time, provide more flexible working hours, increase job satisfaction, and improve work-life balance. However, little is yet known about some of the more fundamental consequences of WFH, including its effects on satisfaction and which factors play a role in making WFH more or less productive than WFO.

In this paper I have provided an analysis of the effects of WFH in T services company. The company abruptly switched all employees from WFO to WFH in March 2020, in response to the largely unanticipated pandemic shock.

The industry and occupations analyzed here are among those predicted to be most amenable to WFH. The employees are highly-skilled professionals in an information technology company where a high degree of work has always been computer driven. At the same time they are some of the most difficult to analyze. The jobs involve significant cognitive work, collaboration on teams, working with clients, and innovation. Satisfaction is hard to measure for such professions. WFH for occupations with such characteristics has not previously been studied with non-survey data.

WFH also affected working patterns in substantial ways. Employees spent more time participating in various types of meetings, but less time in personal meetings with their manager or receiving coaching. They engaged in fewer contacts with colleagues inside and outside of the firm. At the same time, they had less "focus time," i.e., uninterrupted time to perform tasks. All of these factors were significantly correlated with the satisfaction changes due to WFH. These were not temporary adjustments to a switch to WFH, but persisted over time. These findings suggest that increased coordination costs during WFH at least partially explain the drop in satisfaction.

While WFH is likely to remain a feature of modern workplaces, some aspects of in-person interactions cannot easily be replicated virtually including the quality of collaboration and coaching, and "productive accidents" that arise from spontaneously meeting people.

The setting for this study is one of the world's largest IT services companies, with over 150,000 employees who work with clients across the globe. While it employs workers in many countries, the group studied here are employed at its corporate campuses in the home country, a rapidly developing Asian nation. The company provides a wide range of technology consulting and outsourcing services.

The workforce is highly skilled and educated. Virtually all have at least a bachelor's degree, often in a technology field such as computer engineering or electronics. Most work at the company's large, modern corporate campuses in several cities of its home country. These campuses look and feel very similar to what one sees at Microsoft, Apple or Amazon.

The objectives of the research is as follows

- $\quad$ To understand the impact on satisfaction at WFH

- $\quad$ How to achieve Work-Life balance at WFH

- $\quad$ To know the Benefits of WFH to the employees

- $\quad$ To know the drawbacks of WFH to the Organisations \& Employees 


\section{DATA ANALYSIS}

Workplace Analytics Data

Microsoft Workplace Analytics [WPA] is a tool that many companies use to track and analyze various aspects of their workforces .For example, it can be used to study collaboration or professional net-working activity by using data on emails, calendar appointments, amount of time spent in meetings, etc. WPA data have been used in several organizational studies. The company has been considering adoption of this tool. For the purposes of this study they purchased 914 licenses to apply to a subset of employees in our full sample. Appendix Table compares those in the WPA sample to those not in the WPA sample. The WPA group are slightly younger,havelowertenureandarelessproductive, butareoverallquitesimilaronaverage.

\section{Table no 1}

\begin{tabular}{|c|c|c|c|c|c|}
\hline & Mean & SD & 1stQuartile & 3rdQuartile & $\mathrm{N}$ \\
\hline \multicolumn{6}{|c|}{ WFO(beforeMarch15th2020) } \\
\hline WorkingHours & 44.71 & 5.16 & 43 & 46.46 & 6755 \\
\hline AfterHours & 9.64 & 9.55 & 2.33 & 14.04 & 6755 \\
\hline FocusHours & 34.49 & 9.02 & 30 & 41.25 & 6755 \\
\hline CollaborationHours & 10.20 & 9.24 & 3.55 & 13.75 & 6755 \\
\hline MeetingsManager & 3.97 & 4.35 & 0.5 & 5 & 6755 \\
\hline Meetings 1:1 & 0.18 & 1.37 & 0 & 0.5 & 6755 \\
\hline CoachingMeets & 0.13 & 1.03 & 0 & 0 & 6755 \\
\hline MSTeamsCalls & 0.36 & 1.63 & 0 & 0 & 6755 \\
\hline InternalNW & 18.91 & 14.21 & 10 & 24 & 6755 \\
\hline ExternalNW & 2.58 & 3.61 & 0 & 3 & 6755 \\
\hline NWEXT & 0.98 & 1.04 & 0 & 1 & 6755 \\
\hline NWORG & 0.05 & 0.22 & 0 & 1 & 6755 \\
\hline Emails & 23.61 & 23.68 & 9 & 30 & 6755 \\
\hline \multicolumn{6}{|c|}{ WFH(afterMarch15th2020) } \\
\hline WorkingHours & 49.03 & 7.58 & 45.14 & 52.49 & 19220 \\
\hline AfterHours & 12.98 & 12.70 & 3.71 & 18.44 & 19220 \\
\hline FocusHours & 32.73 & 9.99 & 28 & 40 & 19220 \\
\hline CollaborationHours & 11.07 & 9.97 & 4.08 & 15 & 19220 \\
\hline MeetingsManager & 5.48 & 6.57 & 1 & 7.33 & 19220 \\
\hline Meetings 1:1 & 0.11 & 1.07 & 0 & 0 & 19220 \\
\hline CoachingMeets & 0.09 & 0.98 & 0 & 0 & 19220 \\
\hline MSTeamsCalls & 21.46 & 25.22 & 3 & 30 & 19220 \\
\hline InternalNW & 23.44 & 19.89 & 11 & 30 & 19220 \\
\hline ExternalNW & 3.05 & 4.36 & 0 & 4 & 19220 \\
\hline NWEXT & 0.91 & 0.89 & 0 & 1 & 19220 \\
\hline NWORG & 0.05 & 0.23 & 0 & 0 & 19220 \\
\hline Emails & 25.26 & 29.89 & 8 & 30 & 19220 \\
\hline
\end{tabular}

\section{CONCLUSION}

In this paper it has presented the most detailed analysis of WFH satisfaction changes for knowledge workers. The paper makes a number of significant contributions. We study an occupation that is expected to be amenable to WFH, but involves significant cognitive, collaborative and innovation tasks. The data provide an unusually high quality measure of employee satisfaction for knowledge workers. The breadth of the data allow for the first thorough analysis of determinants of WFH satisfaction. We provide evidence on how WFH satisfaction varies with employee characteristics, presence of children at home, and WFO commute time. We also use detailed data on how employees spend their work time to study the effects of job characteristics on WFH satisfaction.

These latter results are important, since they provide insights into how the effectiveness of WFH may vary, and key issues for firms to consider in deploying WFH. 


\section{DOI: $10.17148 /$ IARJSET.2021.81110}

In the sample, employees were able to maintain similar or just slightly lower levels of output during WFH. In order to do so, they worked longer hours. Despite this, employees had less focus time to perform tasks, and the net effect was a drop in satisfaction. It would be interesting to see if this change is sustainable, especially in light of evidence of the adverse effect of long work hours on employee well-being, and mental and physical health (Sparks et al., 1997; Sokejima and Kagamimori,1998;Sparksetal.,2001).

It is likely that WFH also resulted in a decline in intangibles that are valuable to the employee and Company. Working relationships, professional networks and corporate culture may have suffered. More subtly, when people work in the same location, they experience unplanned interactions. That can lead to new working relationships, and "productive accidents" that spur innovation. It is not easy to generate similar unplanned interactions on teleconferences. Finally, employees had fewer opportunities for coaching, and meeting directly with supervisors. This undoubtedly slowed their development of human capital.

We explored several possible explanations for the decline in satisfaction.Our main explanation is that some aspects of work are more difficult to perform in a virtual environment. We provide clear evidence that this is the case. More time was spent in meetings, and those tended to involve larger groups. Less time was spent in direct interactions with the supervisor or colleagues. Employees also narrowed their spheres of communication, interacting with fewer people and business units, both inside and outside the firm. Collectively these indicate that costs of communication, collaboration and coordination are higher when done virtually. Moreover, these factors are likely causes of changes in focus time, and in the decline in satisfaction.

\section{REFERENCES}

[1] Barrero, J., N. Bloom, and S. Davis (2021): “Why Working From Home Will Stick,” Working Paper, Department of Economics, Stanford University.

[2] Aakvik, A., F. Hansen, and G. Torsvik (2017): "Satisfaction dynamics, performance feedback and group incentives in a sales organization," Labour Economics, 46, 110 - 117.

[3] Bartel, A., C. Ichniowski, and K. Shaw (2007): "How does information technology affect satisfaction? Plant-level comparisons product innovation, process improvement, and worker skills," The Quarterly Journal of Economics, 122, 1721-1758. 